semiconductor conference in Paris does show some quantitative changes.

After an introductory chapter in which the history and crystallography of the III-V compounds were discussed, the author discusses band theory as it applies to these substances. This is supplemented by some brief remarks concerning the chemical binding which emphasize the persistent failure of the 'band' and 'bond' pictures to coalesce. The review given in this chapter is of considerable value though in the case of the k.p. theory it would have been better if either less detail or more explanation had been given.

The chapter on optical properties is probably the best in the book. It consists of a review of optical methods of studying band structure, with illustrations drawn from the III-V compounds, and is to be recommended to anyone interested in understanding how these techniques are used. The degree of sophistication and subtlety in experimentation and interpretation is very well brought out; by comparison the picture derived from the transport properties lacks precision. It is only where a detailed numerical calculation has been carried out that one feels confident in the analysis of the experimental data. This situation probably explains why the chapter on transport properties has much more of the character of a compilation than that which precedes it. The same is almost inevitably true of that dealing with mixed crystals and ternary compounds.

The subject of impurities and defects must have been difficult to deal with, since it is in this area that many of the unsolved problems crop up. Consequently there are probably more controversial assertions here than elsewhere, though it seems to me that fair treatment has been given to conflicting points of view.

The survey of $p-n$ junction phenomena is probably the place at which the timing of the book was rather unfortunate owing to the discovery of the diode laser. Only the briefest account of this subject is given, and it will certainly bulk larger in any subsequent edition Prof. Madelung may bring out.

There is a very complete bibliography up to the beginning of 1964 and the division into subject and substance indexes is useful. The translation only rarely reveals that the book was originally written in German and is only positively infelicitous on the dust jacket. This book should prove of great value to all those interested in the physics of III-V semiconductors.

J. E. PARROTT

\section{ASSESSMENT OF OPTICAL SYSTEMS}

\section{Fourier Methods in Optical Image Evaluation}

By Dr. E. H. Linfoot. (The Focal Library.) Pp. 90. (London and Now York: The Focal Pross, 1964.) 50s.

THE assessment of optical systems has, traditionally, been predominantly by spot diagrams derived from ray-traces. That of photographic emulsions has been in terms of subjective 'graininess' and 'limiting resolution'. These means are no longer adequate for the assessment of modern high-performance optical systems.

Even if the spot-diagram fully represented the optical performance, its interpretation would remain ambiguous; it is not evident whether a concentrated core surrounded by a relatively weak but extended halo is better or worse than a comparatively uniform spot-distribution of moderate size. In fact, of course, the spot-diagram distribution does not represent the image structure even qualitatively when the aberration-spreads and diffractionspreads become comparable, since the diffraction-spread corresponding to an aberrant zone (Babinet's principle) may then exceed the whole ray-theoretic image spread.

The fact that almost all images of interest are eventually presented to the human retina provides some justification for the subjective assessments traditionally used in photography. It has become increasingly clear, however, that 'graininess' and 'limiting resolution' are not well defined either conceptually or operationally, and they do not obey any practicable calculus.

Finally, the assessments traditionally applied to optical systems and to photographic emulsions have been mutually isolated. There has been no means of combining spot-diagrams, diffraction-spreads, and photographic characteristics of noise and resolution into an overall assessment of a photographic optical system.

In recent years, these difficulties have been overcome by the application of the methods of communication theory, and their further development for optical applications. Because of the wave-nature of light, an optical image is effectively band-limited, and because of the particle-nature of light the image has a finite signal-tonoise ratio. It follows that the image has a finite information content. Roughly speaking, this means that it is capable in principle of distinguishing one out of a finite set of object intensity-distributions. Useful assessments can be formulated in terms more or less closely related to the extent to which a practical optical system approaches the theoretically possible discriminating-power corresponding to its size and the amount of available light.

The basic analytical tool for these assessments is the Fourier theory. The object-and image-distributions are analysed into their orthogonal sinusoidal components. These components are propagated through an optical system with (in a useful approximation) change only in amplitude and phase; this is a much simpler situation than that for point-components of the object distribution, which may change shape in a complicated manner. The performance of the whole system can be expressed completely (in this approximation) by specifying, as a function of frequency of the sinusoids, the change in signal-to-noise ratio and contrast between object and image distributions. The treatment is implicitly wave-theoretic, although rayapproximations can be derived in some circumstances; photographic effects are included. From these full descriptions it is possible to abstract figures of merit, each of which may be expected to be especially useful in particular circumstances; in particular, it is possible to specify the performance in relation to a prescribed objectset. It turns out that most of the intuitively satisfactory abstractions are closely related, so that it is seldom necessary to make a decision between them.

Dr. Linfoot has played a leading part in these developments, and his book provides a welcome exposition of the basic theory, and of some illustrative results, within the pages of a single compact volume. A careful balance has been maintained between the mathematical and physical content; the basie Fourier theory is collected in an appendix, and the whole treatment requires for its understanding no mathematical technique beyond elementary analysis. After a preliminary chapter exposing the problems and intentions, the processes occurring in optical and photographic imaging are treated by Fourier methods. This leads up to quality assessments of optical and photographic images, and numerical results are given illustrating the interaction between wave aberrations and photographic spread in some selected special cases. The general theory is equally applicable to receiving surfaces other than photographic, and in particular to photoelectric image devices.

The standard of production of the book is high. The paper and layout appear excellent, and the type-face untiring to read. The price works out at about sixpencehalfpenny per numbered page. Since this is more than the cost of duplication on the best present-day office copiers, it raises the question of what part printing ought to play in the dissemination of scientific knowledge to-day. This is not, of course, a reflexion on the merit of the book itself, which can be confidently recommended.

P. FELLGETT 